\title{
Analysis of Eye Gazing on Read Aloud and Problem Solving Activities
}

\author{
Kyota Aoki, Shinjiro Murayama, and Kohji Harada
}

\begin{abstract}
Recently, we can use cheap and easy to use eye gazing measurement tools. However, these tools generate large amount of data that represent the gazing points. This paper proposes a method to analyze the eye gazing data on reading aloud and problem solving activities. The proposed method finds irregular pupils. The algorithm and the experimental results are proposed.
\end{abstract}

Index Terms - Eye gazing, reading activity, problem solving, automatic analysis.

\section{INTRODUCTION}

Teachers observe the activities of pupils, and know the types and the features of them. Teachers teach the pupils based on the knowledge. However, the observations must be subjective and non-quantitative. These types of knowledge are difficult to generalize.

Reading is one of important parts of human activities. Reading ability is one major theme in education. Reading ability is a base to learn many subjects.

In Japan, if a pupil shows two years' delay of reading ability, the pupil is regarded as to have a reading difficulty. Some Japanese normal public elementary schools have about $20 \%$ of pupils with a light reading difficulty [1]. Of course, there are pupils with a heavy reading difficulty. The pupils with a heavy reading difficulty attend special support education classes or schools. In primary school years, girls show about two years progress than boys in their development. This difference makes it difficult to teach boys and girls in a same class [2].

To help a pupil, an ICT device must understand the activity of a pupil precisely. A human teacher can observe and understand not only the activity but also the inner state of a pupil. However, it needs a huge computation power and a huge measuring system.

Text reading prosody and reading comprehension have relations with each other [3]. Teachers observe a pupil to read aloud a text, and understand the ability of the pupil about text reading prosody. With some questions or tests, teachers estimate the reading comprehension of the pupils about the text. For understanding the features of a pupil, teachers need to know the abilities about text reading prosody and reading comprehension.

Japanese texts include many kinds of letters: Hiragana,

Manuscript received January 13, 2019; revised May 19, 2019.

Kyota Aoki and Khoji Harada are with Utsunomiya University, Utsunomiya, Japan (e-mail: kyota@is.utsunomiya-u.ac.jp kharada@utsunomiya-u.ac.jp).

Shinjiro Murayama is with University of Tsukuba, Tokyo, 112-0015 Japan (e-mail: cjc42970@ams.odn.ne.jp).
Katakana, and Kanji. Therefore, reading Japanese texts may have many types of difficulties. We have proposed the system to observe reading activities using face images and read aloud voices [4]. The system can observe the activities about reading aloud, and generate an objective and quantative report about text reading aloud. However, the system cannot measure the precise gazing positions in the reading activities. Recently, we can use cheap and easy to use eye gazing detection devices. With the device, precise gazing detection is possible [5]. Precise observation of reading and problem solving activities is enable now [6]. With the eye gazing detection devices, a system to observe reading activities and problem solving activities with eye gazing detection is implemented [7], [8]. The system generates large amount of eye gazing data. The eye gazing data itself is helpful to understand the pupils' reading and problem solving activities. However, the large amount of data prevents to analyze precisely in normal Japanese primary schools.

This paper proposes a method to analyze objective observations of pupils about their reading aloud and problem solving activities with eye gazing detection to find pupils that teachers need more precise analysis. Eye gazing data of all pupils in a class are too large to analyze and understand precisely by a teacher.

This paper proposes a method to reduce the amount of eye gazing data to analyze easily by teachers, and calculate the normality measure that represents the degree of normality of a pupil about reading and question answering activities.

First, the reading and problem solving activity observation system is introduced. Then, basic analysis and reduction of the data obtained are discussed. Next, analysis and reduction of eye gazing data is discussed. Then, a normality measure in a group is introduced, and the features of the normality measure is discussed in experiments. And last, we conclude this work.

\section{OBJECTIVE OBSERVATION OF READING AND PROBLEM SOLVING ACTIVITIES}

\section{A. Observation System}

The outline of the observation system is shown in Fig. 1. The system is composed of test presentation, questions and choices presentation, user's activity handing and eye gazing recording sub-systems. All sub-systems operate simultaneously. They keep precise system time.

\section{B. Trial}

One trail is constructed from three stages. The first stage is calibration of a gaze detection device. This stage needs about three minutes to complete. The second stage is a practice reading aloud and problem solving. This stage includes two 
sub-stages. The first one is a text reading sub-stage. The other is a problem solving sub-stage. In this stage, a subject learns the key operations and mouse operations on reading aloud and problem solving with small text and a sample problem under the guidance of a teacher. In the last stage, a subject carries out reading a text aloud and problem solving by itself. This stage also includes two sub-stages as the second stage. In the text reading sub-stage, a sentence read is highlighted as Fig. 2. With the key operation of a subject, the highlighted part moves to the next sentence. The text read includes nine sentences. In the problem solving sub-stage, all sentences of the text read are proposed on the left half of a display, and a question and choice box is proposed on the right half of a display at each question as Fig. 3. The number of question is five.

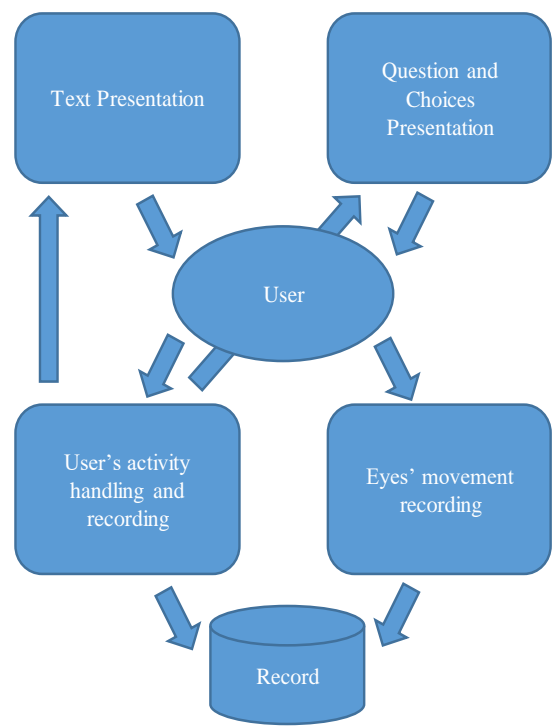

Fig. 1. Observation system overview.

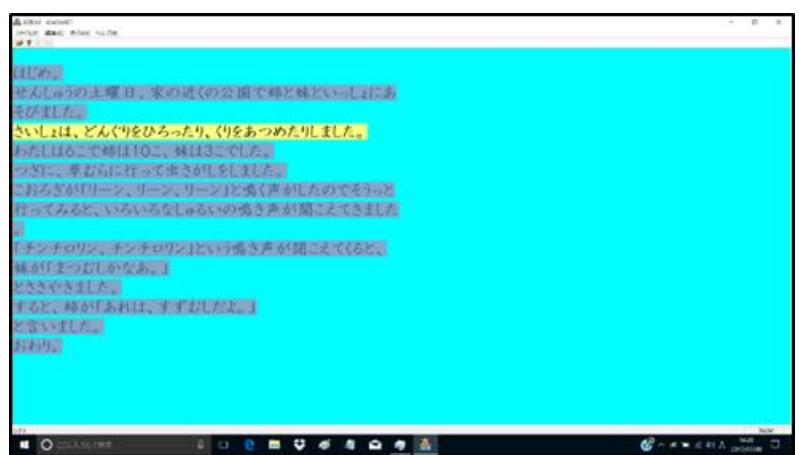

Fig. 2. Example of displayed image on reading aloud process.

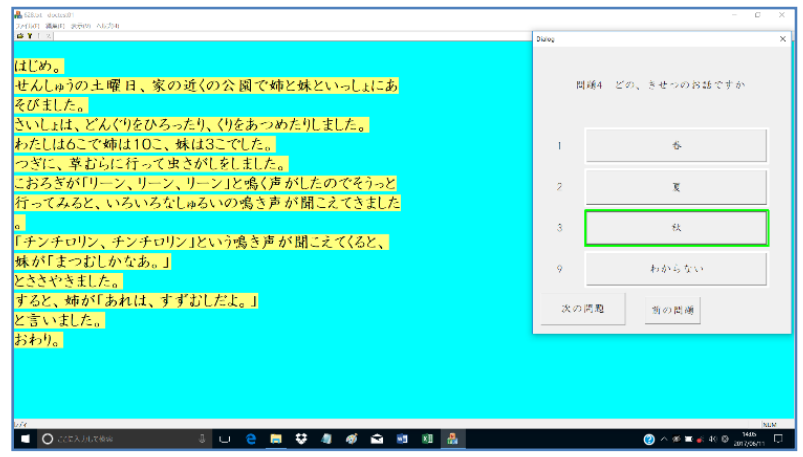

Fig. 3. Example of displayed image on problem solving stage.

\section{Observed Data}

Table I shows the data obtained. In trials, highlighted sentence, key inputs, mouse operations and eye gazing points are recorded with precise time.

TABLE I: THE OBTAINED DATA TyPES AT ACTIVITIES

\begin{tabular}{|c|c|c|c|c|c|}
\hline \multirow[b]{2}{*}{ Stage } & \multirow[b]{2}{*}{ Activity } & \multicolumn{4}{|c|}{ Recorded data } \\
\hline & & $\begin{array}{l}\text { High-lig } \\
\text { hted } \\
\text { sentence }\end{array}$ & $\begin{array}{l}\text { Key } \\
\text { input }\end{array}$ & $\begin{array}{l}\text { Mouse } \\
\text { operation }\end{array}$ & $\begin{array}{l}\text { Eye } \\
\text { gazing }\end{array}$ \\
\hline 1 & $\begin{array}{l}\text { Calibration of } \\
\text { eye gazing }\end{array}$ & $\mathrm{N}$ & $\mathrm{N}$ & $\mathrm{N}$ & $\mathrm{N}$ \\
\hline \multirow[t]{2}{*}{2} & $\begin{array}{l}\text { Practice } \\
\text { reading }\end{array}$ & $\mathrm{Y}$ & $\mathrm{Y}$ & $\mathrm{Y}$ & $\mathrm{Y}$ \\
\hline & $\begin{array}{l}\text { Practice } \\
\text { problem } \\
\text { solving }\end{array}$ & $\mathrm{N}$ & $\mathrm{Y}$ & $\mathrm{Y}$ & $\mathrm{Y}$ \\
\hline \multirow[t]{2}{*}{3} & Reading & $\mathrm{Y}$ & $\mathrm{Y}$ & $\mathrm{Y}$ & $\mathrm{Y}$ \\
\hline & $\begin{array}{l}\text { Problem } \\
\text { solving }\end{array}$ & $\mathrm{N}$ & $\mathrm{Y}$ & $\mathrm{Y}$ & $\mathrm{Y}$ \\
\hline
\end{tabular}

\section{BASIC ANALYSIS OF OBTAINED DATA}

\section{A. Timing of Reading Activities}

The proposed system records the precise time of user's key operation. With the recorded key operations, the reading time of each sentence is measured. From the reading time, we can estimated the reading activity type of a subject.

Fig. 4 shows the display image that shows the relation between a sentence length and a reading time of the sentence. In the left side, some parameters about the diagram are shown. From the diagram, the difficulties of a subject are estimated. The solid line shows the normal reading time of the subject. The normal reading time is estimated removing the outlier data from all measurements.

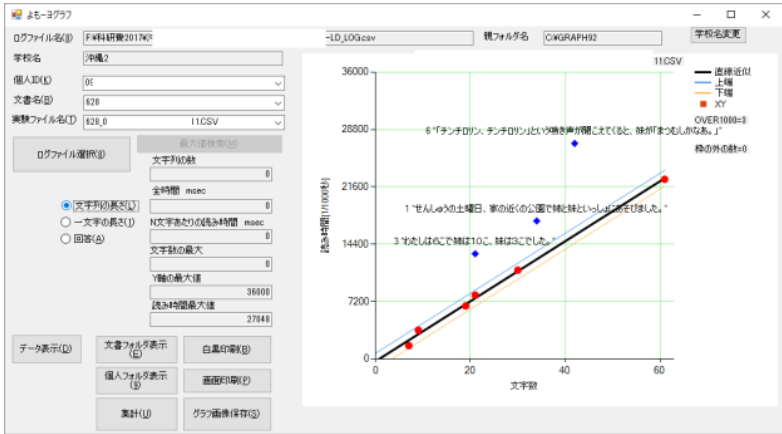

Fig. 4. Example of displayed image of reading time report.

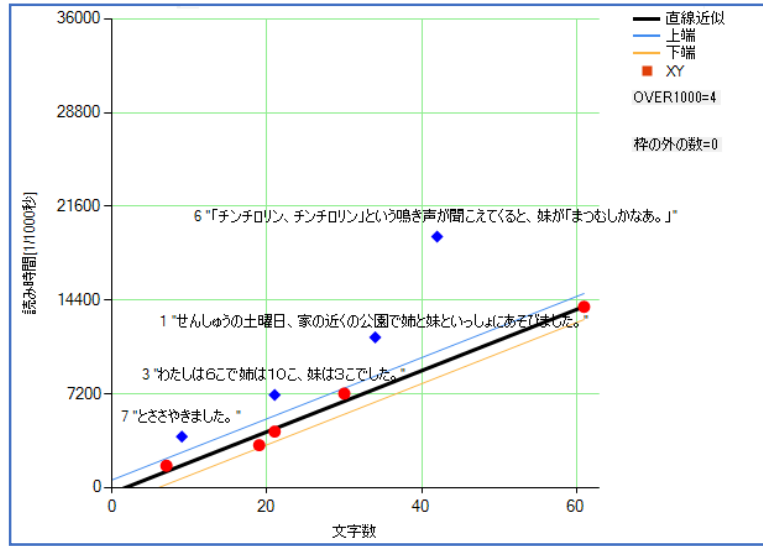

Fig. 5. The relation between reading time and sentence length.

Fig. 5 shows the diagram largely. The horizontal axis is the number of character in a sentence. The vertical axis is the 
consumption time at the sentence in millisecond. In Fig. 5, the outlier sentences are shown with blue diamond marks. A subject has some difficulties to read the sentences. Teachers easily find the sentences that show some difficulties. All of the timings of reading a sentence are proposed on a single diagram. A teacher can look this type of diagrams of all pupils in a class.

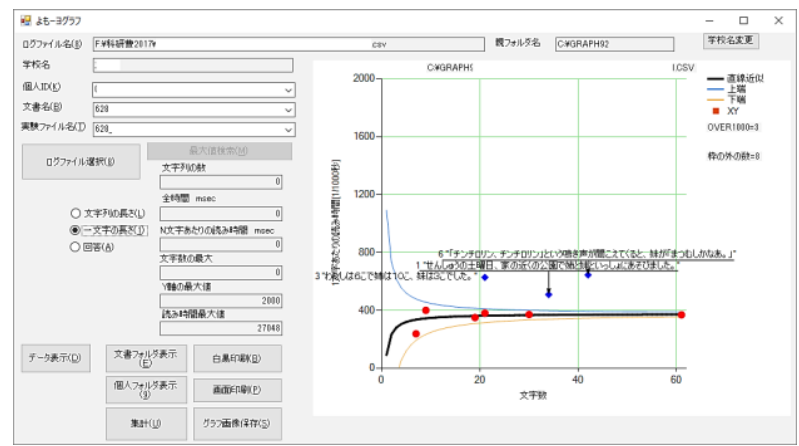

Fig. 6. Example of a displayed image of reading speed report.

Fig. 6 shows the relation between a sentence length and a reading speed of the sentence. From the diagram, other types of reading difficulties are estimated.

\section{B. Timing of Question Answering Activities}

In the question answering stage, the time to answer each question is measured. Fig. 7 is an example of displayed image of question answering report. Fig. 8 shows the diagram largely. In the diagram, answering time of each question is shown. At each question, a tuple of two bars are shown. The left bar shows the time to select an answer. The right bar does the time to proceed to the next question. When an answer is correct, a left bar is blue. Otherwise, the bar is red. In Fig. 8, the subject consumed much time to answer the question 4 , and selected a wrong answer.

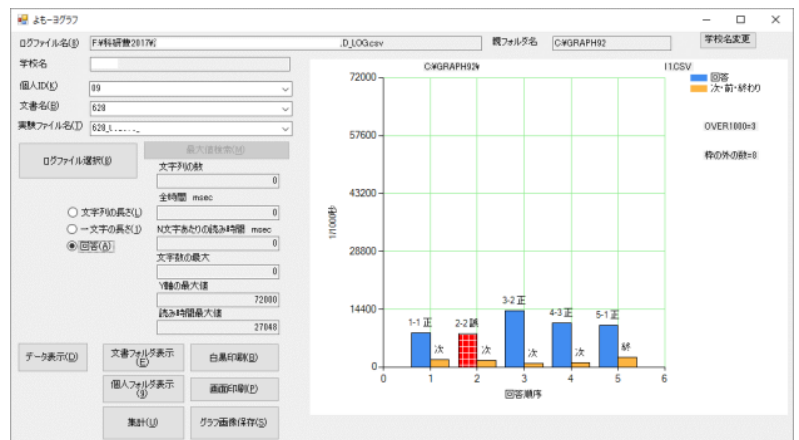

Fig. 7. Example of a displayed image of answering time report.

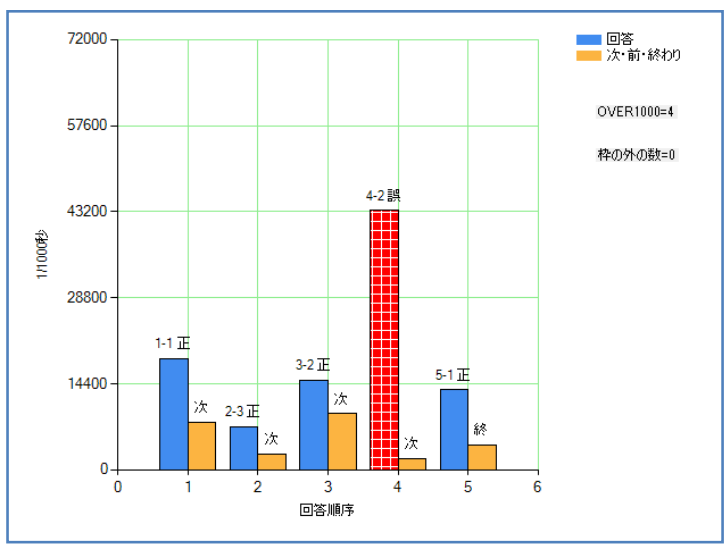

Fig. 8. Question answering time diagram.

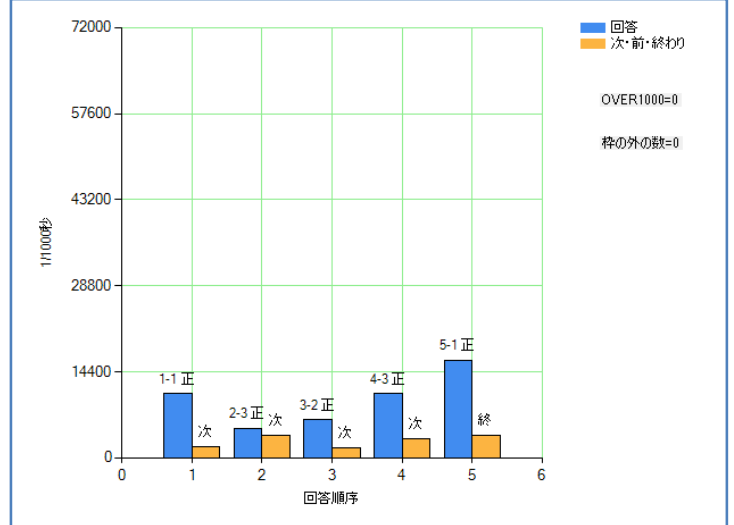

Fig. 9. Question answering time diagram

Fig. 9 shows other example. The subject of Fig. 9 selected all correct answers, and consumed less time than the subject of Fig. 8. With the diagrams, teachers estimate difficulties to understand the text easily and apparently.

This diagram shows all question answering activities. Teachers can look all diagrams of pupils in a class.

\section{Eye Gazing of Reading Activity}

At each sentence, the proposed system makes the tracing of eye gazing. Fig. 10 is an example of the tracing of eye gazing. With this tracing, the reading process of a subject is estimated.

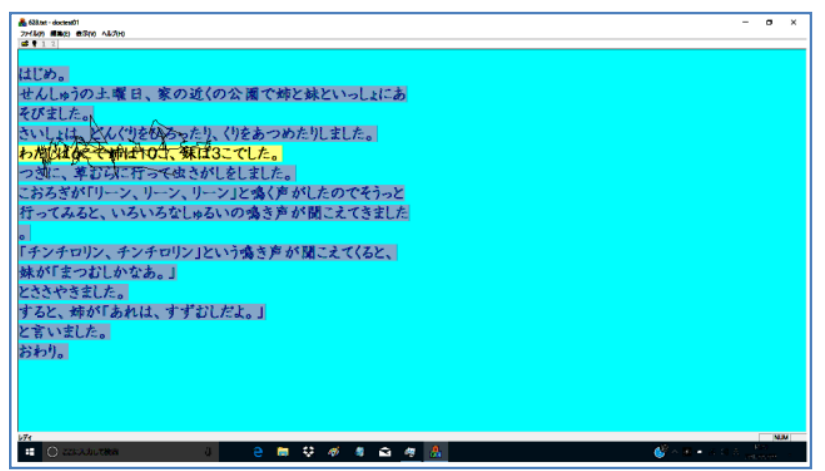

Fig. 10. Example of the movement of eye gazing in a read aloud stage.

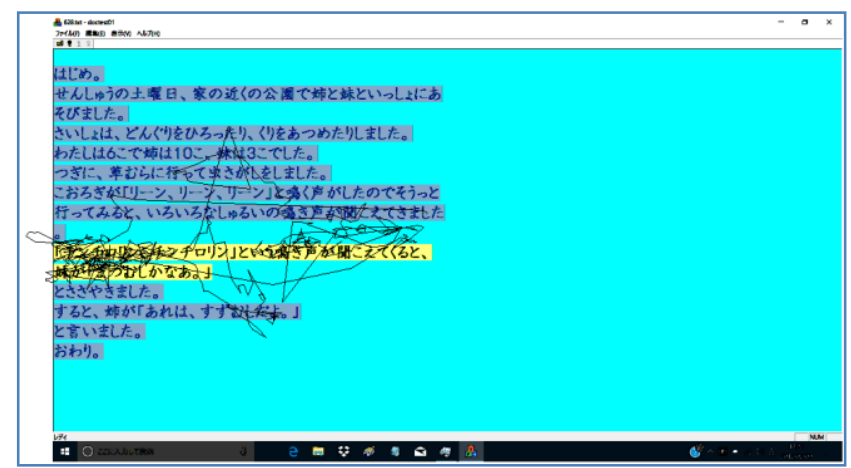

Fig. 11. Other example of the movement of eye gazing in a read aloud stage.

Fig. 11 shows other example. In Fig. 11, the gazing points are distributed on other sentences. The subject of Fig. 11 may have some problem about his eye movements. As this, these eye gazing diagrams show the problems about eye movements clearly. At each subject, nine diagrams are generated. A teacher can look nine diagrams of a pupil. However, at each sentence, this type of diagram is generated. It is difficult to look all diagrams of all pupils in a class. We need to categorize the eye gazing data. 


\section{Eye Gazing of Question Answering Activity}

At each question, the proposed system makes the tracing of eye gazing positions. Fig. 12 is an example of tracing of eye gazing points. Fig. 13 shows other example. With this tracing, the degree of understanding about the text and the activity to search an answer of the question are estimated.

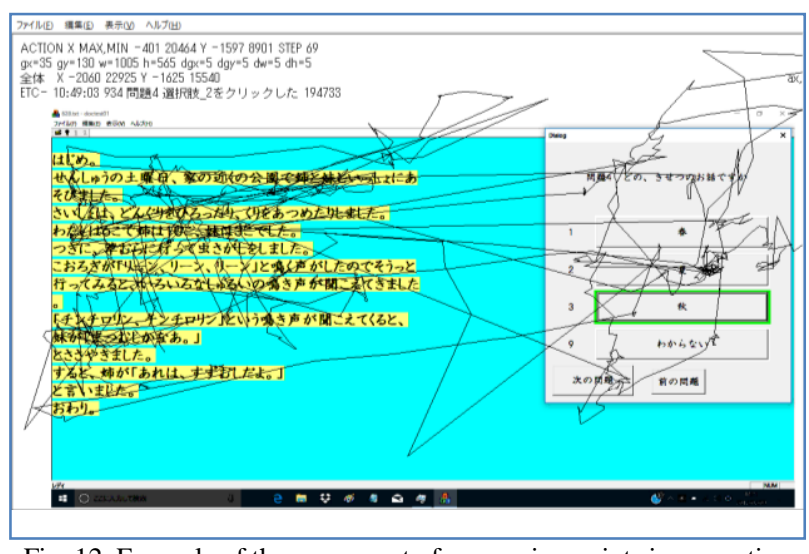

Fig. 12. Example of the movement of eye gazing points in a question answering stage.

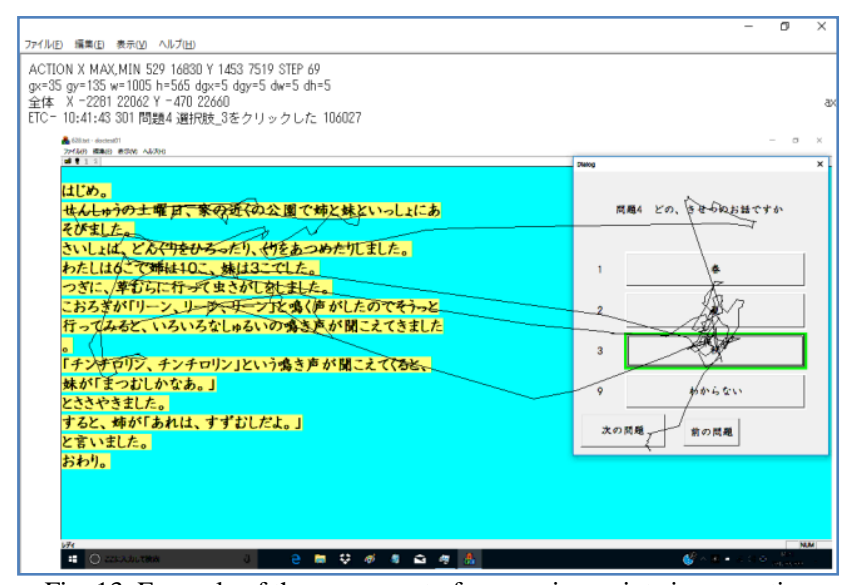

Fig. 13. Example of the movement of eye gazing points in a question answering stage.

The subject of Fig. 12 did not understand the text well. The subject searched the answer through whole text. This subject is estimated not to understand the text well in reading aloud stage.

The subject of Fig. 13 searched only proper sentence. This subject understood the text well in reading aloud stage, and confirmed his understanding. This eye gazing report helps teachers to understand the activities in question answering stage well.

At each subject, five diagrams are generated. A teacher can look five diagrams of a pupil. However, at each pupil, this type of diagram is generated. It is difficult to look all diagrams of all pupils in a class. We need to categorize the eye gazing data automatically.

\section{ANALYSIS AND REDUCTION OF EYE GAZING DATA}

We need a report that represents the total view of a class in a sheet. In other words, we want to have a total data of a subject in a raw of a spreadsheet.

The timing of activities of all subjects is already represented in a sheet. Therefore, we need to reduce eye gazing data of a trial into a single row of a spreadsheet.

\section{A. Eye Gazing Data}

Eye gazing data is a collection of a triple of a time, a horizontal position, and a vertical position. From the operation records of a subject in reading aloud stage, we can know when the reading action and the question answering action are done. With the timing information, we divide the collection of eye gazing data into each sentence and each question.

\section{B. Eye Gazing Data Reduction Method}

At each sentence and question, we make a total view. The view is based on fundamental statistics of the sequence of the eye gazing position. The view includes the mean, the standard derivation of the horizontal and the vertical positions of eye gazing points. However, these fundamental statistics are too small to understand the features of pupils' activities.

A simple precise feature is introduced. The activity at a sentence and a question is divided into two parts that are both of the first and the second halves of each activity. At each half, the amount of movements of eye gazing points are calculated. The movements of eye gazing points are described by horizontal and vertical length of the movements. Therefore, at each half, four parameters are generated. At each sentence and question, eight parameters are generated. A trial includes nine sentences and five questions. As a result, we have 112 parameters about eye gazing on reading a text activity and question answering activity.

\section{EXPERIMENTS AND RESULTS}

\section{A. Experiment}

The proposed data reduction method was implemented with Python script. The measuring experiments have 153 valid trials. All subjects are the third year of Japanese primary schools. In the third year of a primary school, some pupils show reading difficulties apparently. The text and the questions used are designed to fit pupils of the third year of Japanese primary schools [8].

The raw result of the experiments includes the timing information, and the length of each sentence used, etc. Therefore, the resulting record of each trial has 299 fields. 69 fields include redundant only data. Therefore, we have 230 fields include valid data. From the raw result, we can have interesting information easily.

\section{B. Relation among Subjects}

Our goal is to find subjects that need some help to overcome their reading difficulties automatically. However, it is difficult now. Therefore, we will find irregular subjects from large number of all subjects to make precise analysis by teachers.

In normal primary schools, the majority of pupils are similar to one another about reading ability. Therefore, we can find the pupils to be made precise analysis by finding irregular pupils. Of cause, an irregular pupil may be excellent about reading ability. That kind of pupils also need precise analysis and special cares.

\section{Method to Find Irregular Pupils}

We introduce the average of correlations between a subject 
and every subject in a group as the normality measure of the subject in the group. A group may be all pupils of same grade of a school, or it may be all pupils of a same grade of many schools as the experiments of this paper. The normality measure is defined as (1) formally.

$$
\mathrm{NM}(a, G)=\sum_{g \in G} \operatorname{correl}(a, g)
$$

In (1), $a$ is a subject. $G$ is a group of subjects. $N M(a, G)$ is the normality measure of a subject $a$ in the group $G$. $\operatorname{correl}(a$, $g$ ) is a normalized correlation between a subject $a$ and a subject $g$ in the group $G$.

$N M(a, G)$ is normalized in the group $G$. The normalized value is used.

Our reduced eye gazing and timing data about reading and question answering activities makes a collection of 230 parameters. The two collections of parameters of similar two pupils are similar. In the case, the correlation of the two collections is large.

In major normal pupils, their correlation of the collections of parameters are large. Between major normal pupils and miner irregular pupils, the correlations must be small.

About major normal pupils, the majority of correlations are large. The minority of correlations are small. Therefore, the total of correlations are large.

In minor irregular pupils, the correlations distribute wide range, because the irregular pupils include many kinds of specialties. If two irregular pupils are similar, the correlation between the two pupils is large. However, many irregular pupils are different each other. Therefore, the correlations of many pairs of two irregular pupils are small. As a result, the total of correlations of a minor irregular pupil is small.

Table II shows the algorithm of calculating the degree of normality.

TABLE II: The Algorithm to CAlculate Normality MeAsure

\begin{tabular}{|c|l|}
\hline step & Process \\
\hline 1 & $\begin{array}{l}\text { Calculate the correlations between all pairs of reduced } \\
\text { collections of parameters of pupils. }\end{array}$ \\
\hline 2 & Normalize every correlations at each parameter. \\
\hline 3 & $\begin{array}{l}\text { Calculate the total of the normalized correlations at each } \\
\text { pupils. }\end{array}$ \\
\hline 4 & Normalize the totals. \\
\hline
\end{tabular}

The resulting normality measure represents the degree of normality of a subject in a group. If this normality measure is positive, the subject is normal. If the measure is negative, the subject is irregular. This includes the cases that a subject is excellent and has difficulties.

\section{Experimental Result and Discussions}

TABLE III: THE NUMBER OF PUPILS UNDER CUTOFF

\begin{tabular}{|c|c|c|}
\hline Cutoff & Number & $\%$ \\
\hline-3 & 3 & 2.0 \\
\hline-2 & 9 & 5.9 \\
\hline-1 & 22 & 14.4 \\
\hline 0 & 50 & 32.7 \\
\hline 1 & 147 & 96.1 \\
\hline
\end{tabular}

Fig. 14 plots the normality measure of all subjects in ascending order. In the experimental group, normal pupils are majority. Therefore, many subjects show the positive normality measure values. The area of positive normality measure shows small inclination.

Table III shows the number of pupils their normality measure is less than the cutoff value. The number of pupils that normality measure is less than -1 is only 22 . This is $14.4 \%$ of total subjects. Teachers understanding says that about $20 \%$ of pupils in a normal class have some reading difficulties.

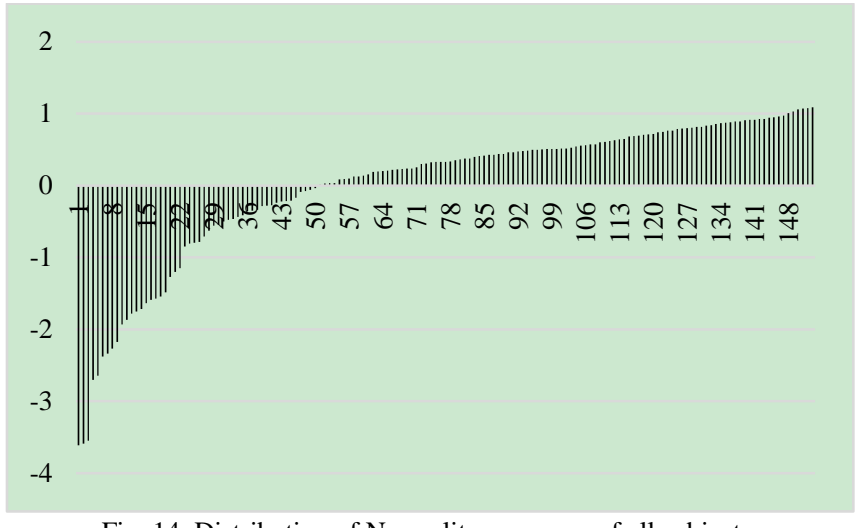

Fig. 14. Distribution of Normality measures of all subjects.

\section{CONCLUSION}

This paper proposed the method to analyze the eye gazing data at reading activity and question answering activity. The proposed normality measure is a simple scaler value, and is easy to use. The measure helps teachers to find the irregular pupils about reading and question answering activities. Irregular pupils may be excellent or have difficulties about reading. In both cases, teachers need to make precise analysis and cares.

\section{ACKNOWLEDGMENT}

This work is supported by JSPS KAKENHI 16K04818 and $16 \mathrm{~K} 01057$.

\section{REFERENCES}

[1] Y. Yaguchi, S. Kotaka, and N. Kajii, "2015. Characteristics of verbal abilities of children with peripheral developmental dyslexia," The Science of Reading, vol. 57, no. 3-4, pp. 47-54, Oct. 2015.

[2] K. Aoki, N. Fukuda, and H. Hotta, "Gender difference of developments of motor control function in primary school ages with visual synchronization task of hands' movement," Inter. Journal of Social Science and Humanity, vol. 7, no. 5, pp. 269-273, May 2017.

[3] N. J. Veenendaal, A. G. Margriet, and L. Verhoeven, "Bidirectional relations between text reading prosody and reading comprehension in the upper primary school grades: A longitudinal perspective," Scientific Studies of Reading, vol. 20, no. 3, pp. 189-202, 2016.

[4] K. Aoki, S. Tashiro, and S. Aoki, "Precise estimation of reading activities with face image and read aloud voice," in Proc. the International Conference on Computer Supported Education 2017, pp. 315-322, April 2017.

[5] D. Rivas-Lalaleo, V. Luna, M. Alveriz, V. Andaluz, W. Quevedo, A. Santana, G. Vayas, M. Navas, and M. Huerta, "System of evaluation for reading based on eye tracking," SETE 2017, LNCS 10676, pp. 234-241, 2017.

[6] K. Aoki, S. Murayama, and K. Harada, "Automatic objective assessments of Japanese reading difficulty with the operation Records on Japanese text presentation system," in Proc. 6th International Conference on Computer Supported Education, vol. 2, pp. 139-146, April 2014.

[7] EyeX. [Online]. Available: https://help.tobii.com/hc/en-us/articles/212818309-Specifications-forEyeX

[8] K. Aoki, S. Murayama, and K. Harada, "Objective observations of read aloud and problem solving activities with eyes' movements". 
Copyright $(92019$ by the authors. This is an open access article distributed under the Creative Commons Attribution License which permits unrestricted use, distribution, and reproduction in any medium, provided the original work is properly cited (CC BY 4.0).

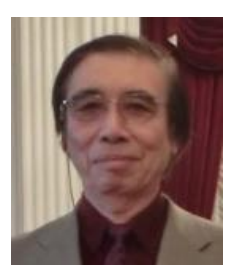

Kyota Aoki was born in Osaka, Japan on December 25, 1951. Kyota Aoki studied Osaka University, before teaching in Utsunomiya with computer science. He had given the $\mathrm{PhD}$ of engineering from Osaka University, Osaka, Japan at 1979. He works in computer science. He started from automaton, and then moved to image processing. He worked at Graduate School of Engineering, Utsunomiya University in Utsunomiya, Japan. He retired from fulltime researcher of Utsunomiya University. Now, he is guest professor of Utsunomiya University. He works for the measurements about human motion and their analysis for the applications in education and health care.

Dr. Kyota is the member of IECIE, IPSJ, JALD, and JSSMID.

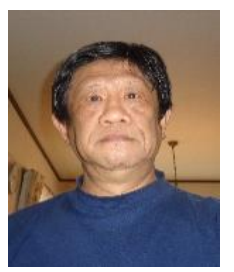

Shinjiro Murayama was born in Tokyo, Japan on April 3, 1956. Shinjiro Murayama graduated from Nihon University School of phisics. He worked at the Institute of Japanese Union of Scientists \& Engineers from 1980. As a lecturer at Special Needs Education School for the Visually Impaired, University of Tsukuba from 2014, and Mashiko special needs school from 2018 , he is conducting research on development of screen reader software for visually impaired people, development of document presentation system for students with disabilities, and survey of persons with disabilities who read and write using gaze measuring devices. Mr. Shinjiro is the member of JALD.

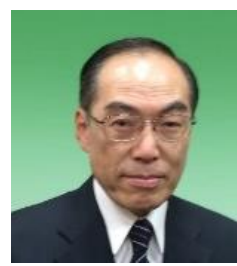

Kohji Harada was born on September 7, 1953. Koji Harada graduated from Doshisha University School of Commerce, and graduated from Utsunomiya University School of Education. He worked as an elementary school teacher since 1980 and served as a primary school principal from 2008. As an associate professor at Utsunomiya University professorial graduate school from 2014, he is conducting research on elementary and junior high school student support method through special support education, developmental disorder, and school management. Assoc. prof. Kohji is the member of JALD 